21st Particles and Nuclei International Conference (PANIC 2017)

International Journal of Modern Physics: Conference Series

Vol. 46 (2018) 1860030 (6 pages)

(C) The Author(s)

DOI: $10.1142 / \mathrm{S} 2010194518600303$

\title{
The High-Energy Photoproduction of Light-Quark Pseudoscalar and Scalar Meson at GlueX
}

\author{
Zhenyu Zhang (For the GlueX Collaboration) \\ Hubei Nuclear Solid Physics Key Laboratory, School of Physics \\ and Technology, Wuhan University, Wuhan, 430072, China \\ zhenyuzhang@whu.edu.cn
}

Published 3 May 2018

\begin{abstract}
The high-energy photoproduction of light-quark pseudoscalar and scalar mesons is an effective tool for understanding the properties of strong interaction in the nonperturbative regime. It has been investigated theoretically using Regge-cut phenomenology with massive quasi-particle exchange in the high-energy regime, and the linearly polarized photon beam asymmetry $\Sigma$ can provide insight into the dominant production mechanism. In the low-energy region, it can provide constraints on "background" to baryon resonance extraction. With an almost 50-year history, intensive experiments on meson photoproduction are growing vigorously at several international laboratories, such as JLab, ELSA, and MAMI. Recently the beam asymmetry $\Sigma$ in high-energy $\pi^{0} / \eta$ photoproduction has been measured at GlueX, which is the first measurement both from the GlueX experiment and the $12 \mathrm{GeV}$ upgraded JLab. The highest precision measurement of the $\pi^{0}$ asymmetry and the first measurement of $\eta$ beam asymmetry at a beam energy above $3 \mathrm{GeV}$ are presented. A broad meson photoproduction project, including scalar meson $a_{0}(980) / f_{0}(980)$, is under way at GlueX. In the proceedings, we report the beam asymmetry results for $\pi^{0} / \eta$ photoproduction at GlueX, as well as preliminary results for scalar meson photoproduction in the $\pi^{0} \pi^{0}$ and $\pi^{0} \eta$ channels.
\end{abstract}

Keywords: GlueX; Photoproduction; Beam asymmetry.

\section{Introduction}

Meson photoproduction has been attracting great interests of researchers in hadron physics recently. Theoretically it can be described by Regge trajectory exchanges at high energy, which has been well understood in pion photoproduction and can also been extend to scalar and tensor mesons reasonably ${ }^{1-4}$.

Using the coherent bremsstrahlung technique, the GlueX Experiment ${ }^{5-6}$ at the 12-GeV-upgraded Jefferson Lab produces a $9 \mathrm{GeV}$ linearly polarized photon beam $^{7}$. The long-term aim of the GlueX experiment is to understand quark-gluon interactions and to search for exotic hybrid mesons, for which the light meson photoproduction is a promising experimental technique. GlueX has been collecting

This is an Open Access article published by World Scientific Publishing Company. It is distributed under the terms of the Creative Commons Attribution 4.0 (CC-BY) License. Further distribution of this work is permitted, provided the original work is properly cited. 
high-energy meson photoproduction data at $9 \mathrm{GeV}$ since the spring of 2016, and a broad meson photoproduction program is under analysis.

Searching for multiphoton final states is a component of a wider meson photoproduction project, which is related to a rich pattern of the physics, such as the nature of pseudoscalar, scalar and tensor mesons, exotic hybrids, dark B boson ${ }^{8}$ searching, and new $\mathrm{C}$ violating, $\mathrm{P}$ conserving reactions. In the proceedings, we focus on $2 \gamma$ and $4 \gamma$ decays for psuedoscalar meson and scalar meson photoproduction.

\section{Psuedoscalar meson $\pi^{0} / \eta$ photoproduction}

The mechanism of psuedoscalar meson photoproduction can be described by the Regge-cut phenomenology through the exchange of the vector or axial-vector Reggeons at leading order ${ }^{9}$. The theoretical approaches on $\pi^{0} / \eta$ photoproduction have been developed and extended recently by several groups ${ }^{1-2,10-12}$. The differential cross sections on the proton target can be written in terms of vector or axial-vector Regge amplitudes,

$$
\frac{d \sigma}{d t}=\sigma_{\perp}+\sigma_{\|}=|\rho+\omega|^{2}+|b+h|^{2}
$$

The beam asymmetry is given by

$$
\Sigma=\frac{|\rho+\omega|^{2}-|b+h|^{2}}{|\rho+\omega|^{2}+|b+h|^{2}},
$$

which is sensitive to the exchange on trajectories of the vector and axial-vector mesons. It will be close to +1 if the process is vector meson dominant, while -1 if axial-vector meson dominant.

The beam asymmetry of $\pi^{0}$ photoproduction off proton at $\bar{E}_{\gamma}=10 \mathrm{GeV}$ has been measured by the Stanford Linear Accelerator Center (SLAC) ${ }^{13}$. The differential cross section as a function of the proton momentum transfer $t=\left(p_{\text {target }}-p_{\mathrm{p}}\right)^{2}$ distribution shows a significant "dip" around $-t=0.5(\mathrm{GeV} / \mathrm{c})^{2}$, where the beam asymmetry also seems to have potential for a "dip". However, the data is sparse and the error bar is large in the area around $-t=0.5(\mathrm{GeV} / \mathrm{c})^{2}$ due to the decrease of the scattering cross section. More precise data is needed to clarify these issues. Besides, there are no previous measurements of the $\Sigma$ asymmetry for $\gamma p \rightarrow \eta p$ with $E_{\gamma}>3 \mathrm{GeV}$. The linearly-polarized photon beam peaking at $9 \mathrm{GeV}$ at GlueX will provide important new constraints on these Regge models.

After the event selections the distribution of the invariant mass of 2 photons is shown in Fig. 1. The peaks of $\pi^{0}$ and $\eta$ are obvious and the continuum background between $\pi^{0}$ and $\eta$ is negligible. MC events for the reaction $\gamma p \rightarrow p \omega, \omega \rightarrow \pi^{0} \gamma$ are simulated to get background shape vs $M_{\gamma \gamma}$ and data is normalized in the $\omega$ control region above the $\eta$ peak. Finally the fraction of $\eta$ yield due to $\gamma p \rightarrow p \omega$ is $\sim 0.38 \%$ and contribution to $\pi^{0}$ yield is negligible.

The $\pi^{0}$ and $\eta$ yields (without corrections for instrument acceptance) as a function of the proton momentum transfer $-t$ is shown in Fig. 2. The distribution for $\pi^{0}$ 


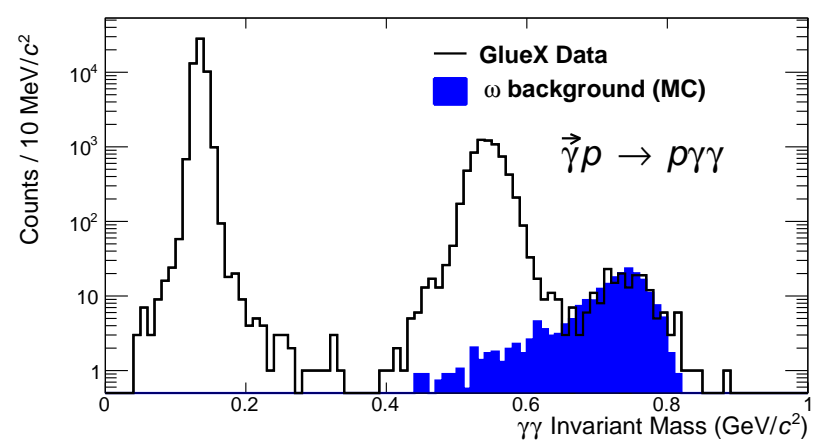

Fig. 1. $\quad \gamma \gamma$ invariant mass distribution with clear peaks at the $\pi^{0}$ and $\eta$ masses, superimposed with background estimated from $\gamma p \rightarrow p \omega, \omega \rightarrow \pi^{0} \gamma$ simulation.

photoproduction shows the expected dip near $-t=0.5(\mathrm{GeV} / \mathrm{c})^{2}$, while the one for $\eta$ photoproduction does not show a dip in the observed $-t$ range. The measurements are consistent with previous measurements in Ref. 13-14.
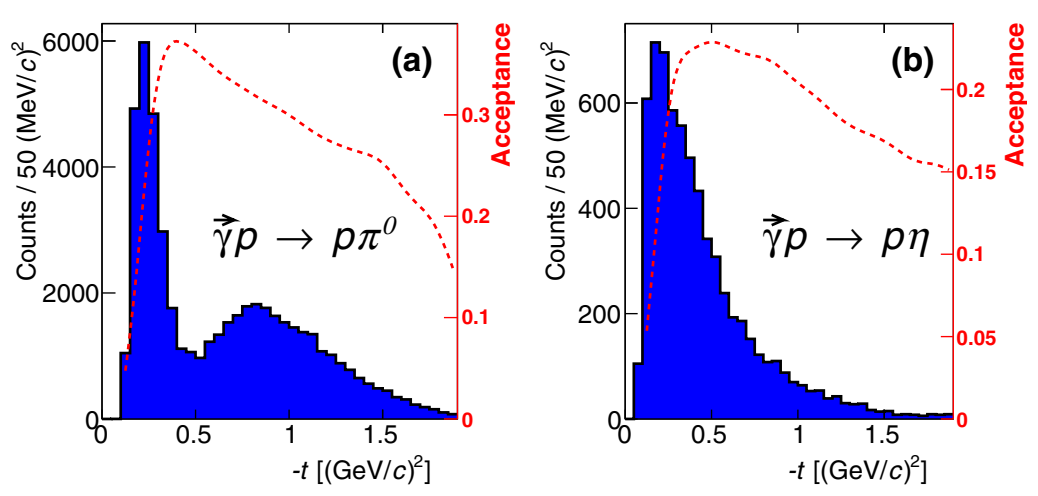

Fig. 2. The $\pi^{0}$ and $\eta$ yields (without corrections for instrument acceptance) as a function of the proton momentum transfer $-t$. The acceptance functions (red dashed), determined from MC simulation, are shown for comparison.

The distribution of the azimuthal angle of the proton production plane $d \sigma / d \phi_{\mathrm{p}} \propto$ $1-P \Sigma \cos 2\left(\phi_{\mathrm{p}}-\phi_{\gamma^{\text {lin }}}\right)$, where $\phi_{\gamma^{\text {lin }}}$ is the azimuthal angle of the beam photon's linear polarization plane, and for PARA $\phi_{\gamma^{\text {lin }}}=0^{\circ}$ and for PERP $\phi_{\gamma^{\text {lin }}}=90^{\circ}$. Here, PARA (PERP) indicate parallel (perpindicular) to the floor, and we collected data in these two conditions. Therefore, the yields for the PERP and PARA orientations are given by

$$
\begin{gathered}
Y_{\perp} \propto N_{\perp}\left(1+P_{\perp} \Sigma \cos 2 \phi_{\mathrm{p}}\right), \\
Y_{\|} \propto N_{\|}\left(1-P_{\|} \Sigma \cos 2 \phi_{\mathrm{p}}\right) .
\end{gathered}
$$



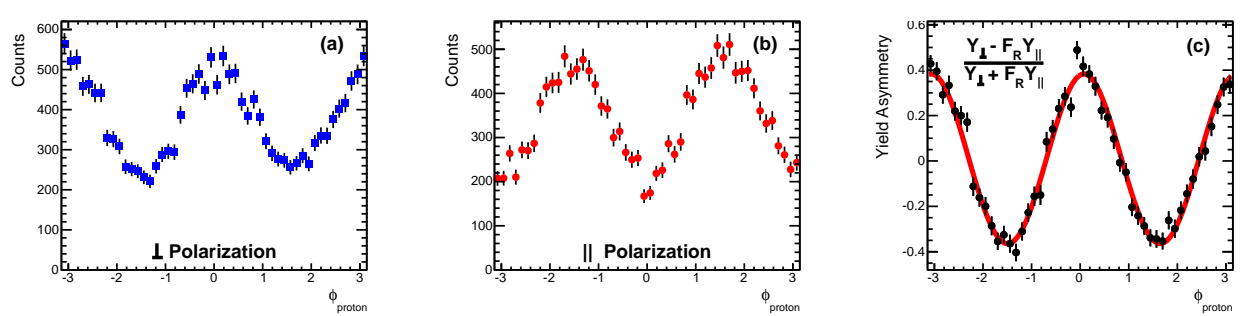

Fig. 3. The yields of $\pi^{0}$ photoproduction verse $\phi_{\mathrm{p}}$ integrated over $-t$. (a) PERP (b) PARA (c) The yield asymmetry fitting with Eq. (5)

After subtracting the background contribution from accidentally tagged photons, the yields for both PARA and PERP are shown in Fig. 3 as a function of $\phi_{p}$. To reduce the instrumental acceptance and the systematic uncertainty, the beam asymmetry $\Sigma$ can be extracted by fitting the azimuthal angle distribution from the expression of the difference of the PERP and the normalized PARA yield over the sum

$$
\frac{Y_{\perp}-F_{R} Y_{\|}}{Y_{\perp}+F_{R} Y_{\|}}=\frac{\left(P_{\perp}+P_{\|}\right) \Sigma \cos 2 \phi_{\mathrm{p}}}{2+\left(P_{\perp}-P_{\|}\right) \Sigma \cos 2 \phi_{\mathrm{p}}},
$$

where $F_{R}=N_{\perp} / N_{\|}$is the ratio of the integrated photon flux between PERP and PARA. The final results are shown in Fig. 4 by repeating the fitting in bins of $-t$ for both $\pi^{0}$ and $\eta$.
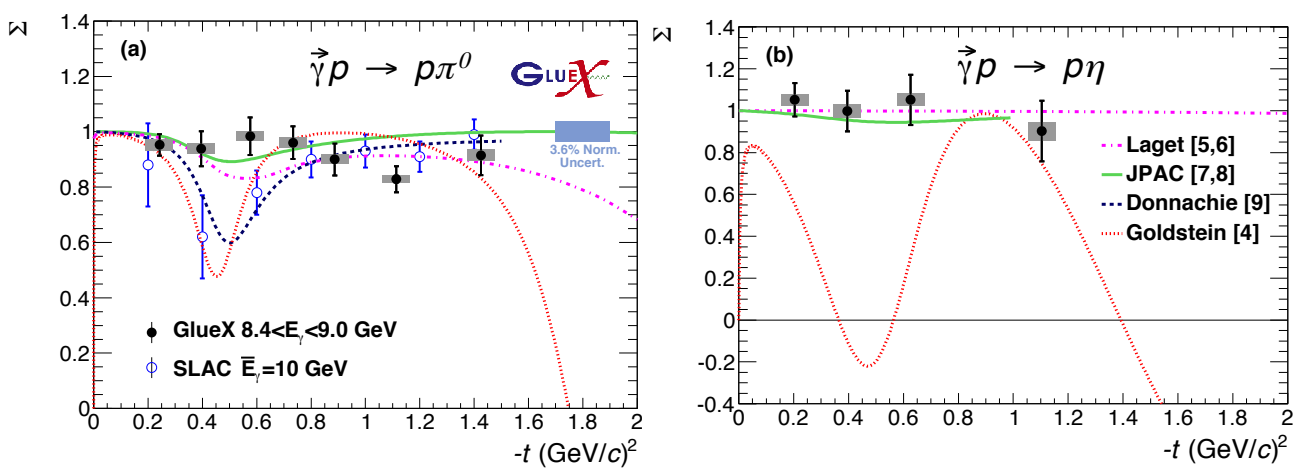

Fig. 4. Beam asymmetry for (a) $\gamma p \rightarrow p \pi^{0}$ and (b) $\gamma p \rightarrow p \eta$ (black filled circles). Uncorrelated systematic errors are indicated by gray bars and combined statistical and systematic uncertainties are given by the black error bars. The previous SLAC results at $\bar{E}_{\gamma}=10 \mathrm{GeV}$ (blue open circles) are also shown along with various Regge theory calculations.

The measured asymmetries $\Sigma$ are consistent with previous SLAC data and close to 1 , with little evidence of $-t$ dependence. The prominent dip in beam asymmetry at $-t=0.5(\mathrm{GeV} / \mathrm{c})^{2}$ isn't observed as predicted by some theory calculations. See Ref. 15 for more details. 


\section{Scalar mesons photoproduction}

The candidate events for $\gamma p \rightarrow p \gamma \gamma \gamma \gamma$ are selected by identifying one tagged beam photon, one positively charged track originating from the target region, and at least four neutral showers in the calorimeters. Fig. 5 shows that in $4 \gamma$ photoproduction, the dominant channels are $\pi^{0} \pi^{0}$ and $\pi^{0} \eta$ channels, which are related to the light scalar mesons $a_{0}(980)$ and $f_{0}(980)$ photoproduction.

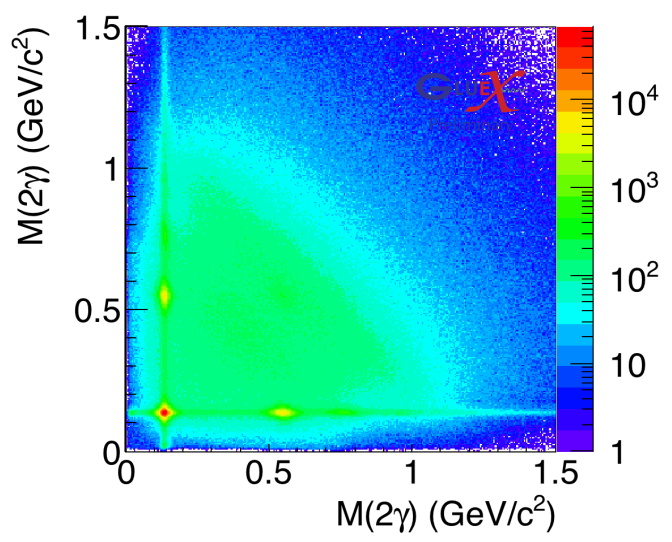

Fig. 5. The scatter plot for $M_{2 \gamma}$ vs $M_{2 \gamma}$ in $4 \gamma$ photoproduction.

Regge-cut phenomenology, which is well understood in pion photoproduction, has been used to study the scalar meson photoproduction ${ }^{2}$. CB-ELSA and CLAS collaborations made some measurements for $a_{0}(980)$ and $f_{0}(980)$ photoproduction respectively at lower energies ${ }^{16-17}$. Published statistics for $a_{0}(980)$ and $f_{0}(980)$ photoproduction is low and in a limited energy range. Fig. 6 shows the two pseudoscalar invariant mass distributions for $\pi^{0} \pi^{0}$ and $\pi^{0} \eta$ photoproduction separately. The contributions from the scalar meson $a_{0}(980)$ can be seen obviously, as well as those from the tensor mesons $a_{2}(1320)$ and $f_{2}(1270)$. However more challenges
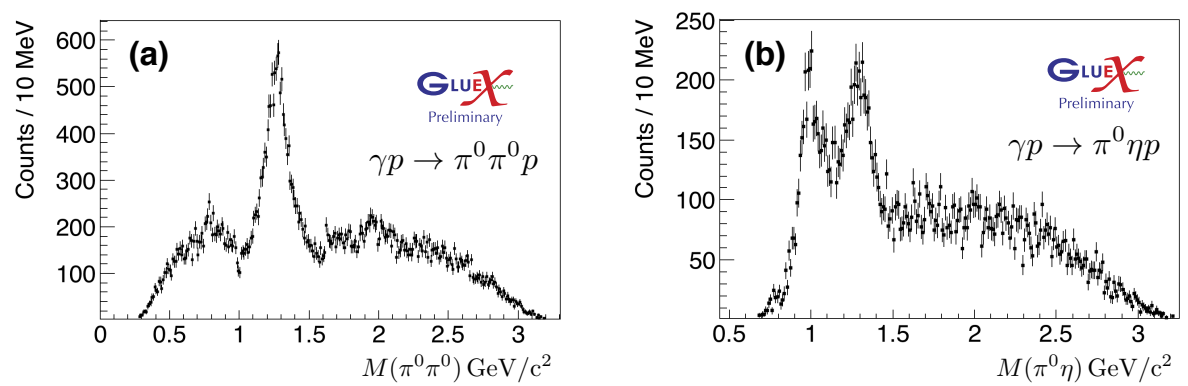

Fig. 6. The invariant mass of two pseudoscalar mesons. (a) $\pi^{0} \pi^{0}$ invariant mass for $\gamma p \rightarrow \pi^{0} \pi^{0} p$; (b) $\pi^{0} \eta$ invariant mass for $\gamma p \rightarrow \pi^{0} \eta p$. 
on the background study exist in these channels than the pseudoscalar photoproduction, among which baryon photoproductions are dominant. To study the scalar meson photoproduction carefully, the moment analysis for di-pseudoscalar-meson photoproduction is underway ${ }^{18}$.

\section{Summary}

A broad meson photoproduction project at GlueX is well under way, including beam asymmetries, cross sections and spin density matrix elements analysis. The linearly polarized photon beam asymmetry $\Sigma$ for $\pi^{0} / \eta$ photoproduction have been measured. A detailed survey of the multi-photon processes is performed deeply. The moment analysis for di-pseudoscalar-meson photoproduction and the beam asymmetry studies of scalar mesons are in progress.

\section{Acknowledgments}

This work is supported, in part, by the National Natural Science Foundation of China (NSFC) under Contract No.11575133, the China Scholarship Council under Contract No.201506275156, and the Hubei Nuclear Solid Physics Key Laboratory. This material is based upon work supported by the U.S. Department of Energy, Office of Science, Office of Nuclear Physics under Contract No. DE-AC0506 OR23177.

\section{References}

1. V. Mathieu, G. Fox, and A. P. Szczepaniak, Phys. Rev. D 92, 074013 (2015).

2. A. Donnachie and Yu. S. Kalashnikova, Phys. Rev. C 93, 025203 (2016).

3. J. Xie, E. Oset, and L. Geng, Phys. Rev. C 93, 025202 (2016).

4. X. Wang, and A. Guskov, Phys. Rev. D 93, 074016 (2016).

5. GlueX Collab. (H. Al Ghoul et al.), AIP Conf. Proc. 1735, 020001 (2016).

6. A. Austregesilo (for the GlueX Collab.) the proceedings.

7. M. Dugger, B. G. Ritchie, and et al., Nucl. Instrum. Methods A 867, 115 (2017).

8. C.Fanelli, M. williams, arXiv:1605.07161 [hep-ph] (2016).

9. G. R. Goldstein and J. F. Owens, Phys. Rev. D 7, 865 (1973).

10. J. M. Laget, Phys. Rev. C 72, 022202 (2005).

11. J. M. Laget, Phys. Lett. B 695, 199 (2011).

12. JPAC Collab. (J. Nys et al.) Phys. Rev. D 95, 034014 (2017).

13. R. L. Anderson, D. Gustavson, et al., Phys. Rev. D 4, 1937 (1971).

14. R. L. Anderson, D. Gustavson, et al., Phys. Rev. D 1, 27 (1970).

15. GlueX Collab. (H. Al Ghoul et al.), Phys. Rev. C 95, 042201 (2017).

16. CB-ELSA Collab. (I. Horn, it et al.), Eur. Phys. J. A 38, 173 (2008).

17. CLAS Collab. (M. Battaglieri et al.) Phys. Rev. Lett. 102, 102001 (2009).

18. Shuang Han (for the GlueX Collab.) Fall Meeting of the APS Division of Nuclear Physics (2017), 62, KK.1, http://meetings.aps.org/link/BAPS.2017.DNP.KK.1. 